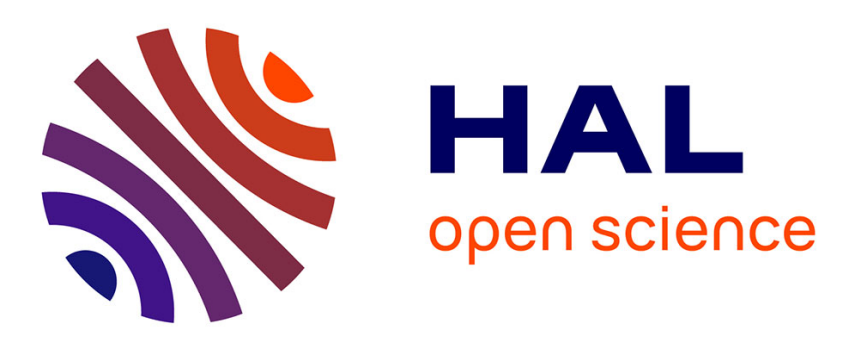

\title{
Paradoxes in Semi-Dynamic Evolutionary Power Control Game: When Intuition Fools You!
}

\author{
Majed Haddad, Eitan Altman, Dieter Fiems, Julien Gaillard
}

\section{To cite this version:}

Majed Haddad, Eitan Altman, Dieter Fiems, Julien Gaillard. Paradoxes in Semi-Dynamic Evolutionary Power Control Game: When Intuition Fools You!. IEEE Transactions on Wireless Communications, 2013, 12 (11), pp.5728-5739. 10.1109/TWC.2013.101613.122041 . hal-00913143

\section{HAL Id: hal-00913143 \\ https://hal.inria.fr/hal-00913143}

Submitted on 10 Dec 2013

HAL is a multi-disciplinary open access archive for the deposit and dissemination of scientific research documents, whether they are published or not. The documents may come from teaching and research institutions in France or abroad, or from public or private research centers.
L'archive ouverte pluridisciplinaire HAL, est destinée au dépôt et à la diffusion de documents scientifiques de niveau recherche, publiés ou non, émanant des établissements d'enseignement et de recherche français ou étrangers, des laboratoires publics ou privés. 


\title{
Paradoxes in Semi-Dynamic Evolutionary Power Control Game: When Intuition Fools You!
}

\author{
Majed Haddad, member, IEEE, Eitan Altman, Fellow, IEEE, Dieter Fiems, member, IEEE and Julien Gaillard
}

\begin{abstract}
This paper studies a power control game over a collision channel. Each player has an energy state and balances energy conservation and transmission success. When opting for higher transmission power, the chances of a successful transmission in the presence of interference increases at the cost of a larger drop in energy. We study this dynamic game when restricting to simple non-dynamic strategies: a power level is chosen at start-up and maintained during the lifetime of the battery. A thorough analysis of the existence and characterization of the equilibria of this evolutionary Hawk-Dove game is conducted. Moreover, we study the stability of our results under various classes of evolutionary dynamics, including replicator dynamics and Brown-von Neumann-Nash (BNN) dynamics and identify various surprising paradoxes. Simulation results validate our theoretical claims.
\end{abstract}

Index Terms-Energy saving, power control, bio-inspired networks, evolutionary game theory, dynamics, paradoxes.

\section{INTRODUCTION}

This paper considers a semi-dynamic variant of the well known Hawk and Dove game [2]. This game was originally introduced to describe the evolution of aggressive behavior amongst animals competing for food but has been applied to entirely different problems as well. For example, variants of the Hawk-Dove (H-D) game have been successfully applied in networking. The medium access game considers competition over access to a common channel through the control of the attempt probabilities [3]. The power control game studies the choice of transmission power over a collision channel [4]. Finally, in congestion control, the H-D game can be used to study the choice between different versions of TCP (transmission control protocol) to be used over the Internet [6].

In the H-D game, there are two types of individuals: aggressive individuals (Hawks, denoted by $H$ ) and peaceful individuals (Doves, denoted by $D$ ). Variants of the H-D game have been successfully applied in networking. The medium access game considers competition over access to a common channel through the control of the attempt probabilities [3]. The power control game studies the choice of transmission power over a collision channel [4]. In congestion control, the

Majed Haddad, Eitan Altman and Julien Gaillard are with INRIA SophiaAntipolis, 10 route des Lucioles, 06902 Sophia Antipolis, France. (e-mail: majed.haddad, eitan.altman, julien.gaillard@inria.fr).

Dieter Fiems is with Department TELIN, Ghent University, St-Pietersnieuwstraat 41, 9000 Gent, Belgium (email: Dieter.Fiems@telin.UGent.b).

Manuscript received December 22, 2012; revised March 31, 2013, accepted August 23, 2013.
Hawk and Dove game can be used to study the choice between different versions of TCP (transmission control protocol) sharing a common bottleneck link to be used over the Internet [5]. In [6], the Hawk strategy stands for the Scalable TCP and the Dove strategy stands for New-Reno TCP.

The literature on optimally controlling transmission power of wireless devices sharing a common medium is considerable. In [7], Yates presents one of the first distributed power control algorithms. In [8], Goodman et al. first proposed an energyefficient power control game for flat fading channels. In this paper, we revisit the power control problem. We use the classical framework of evolutionary games, which we extend to a semi-dynamic context (see below). Such games deal with large populations in which individuals interact with each other through many local interactions, each of which involve two randomly selected individuals. Several previous papers have already studied evolutionary games with pairwise local interactions in the context of wireless networks (see for instance [3], [9], [13]). This pairwise interaction paradigm [10] is relevant for situations of sparse mobile networks in which one may neglect the possibility of simultaneous interference of more than two mobiles.

The standard Hawk and Dove game predicts which type of behavior ( $H$ or $D$ ) will dominate in the long run, and when the coexistence of aggressive and peaceful individuals may be expected. The equilibrium fraction of the two types is obtained by solving a two player auxiliary matrix game. Several authors have studied dynamic variants of this game where individuals are characterized by their energy state [4], [6], [12]-[14]. A biological variant of this game can be found in [15]. Aggressive behavior requires more energy (which is the case in both the MAC problem as well as in the original H-D example). The energy reserve of an individual is defined as the individual state. Thus actions of an individual influence not only the immediate fitness but also the future state of the individual. The objective of an individual consists of maximizing the total expected fitness during its lifetime. In these dynamic versions of the Hawk and Dove game, the individual strategy is no longer a single choice between $H$ and $D$, but rather a collection of choices that prescribes how an individual should behave at each possible state.

We emphasize that the power control context is not a restriction of the proposed Hawk and Dove model. Apart from the fact that they may use either low or high transmit power level, mobile users may have different constraints on their battery. As an example, a small terminal, e.g., dump- 
phones are more likely to be equipped with a small battery and therefore behave as Doves while smart-phones are more likely to behave as Hawks since they may consume more battery. Therefore, depending on their type and the context, mobile terminals will behave as Hawk or Dove where Hawk and Dove stand both for a type and for the power level transmitted at a given time by a given mobile.

We consider a semi-dynamic framework which inherits some features from the static framework and some from the dynamic one. As in the dynamic setting, each player has an individual energy state and the player's action determines not only the immediate fitness but also the distribution of the future energy state. Yet in contrast to the dynamic versions of the game, we assume that an individual makes state independent choices. The individual chooses $i$ (where $i$ is $H$ or $D$ ) and once the choice is made, the same action $i$ is always used by this individual at any state: the individual is either always aggressive or always peaceful. The problem thus resembles the static one in the fact that the individual has to choose only once, between $D$ and $H$.

We shall use the two central concepts of evolutionary games. The first is the concept of an Evolutionary Stable Strategy (ESS), which is a distribution of (deterministic or mixed) actions such that, if used, the population is immune against penetration of mutations [16]-[18]. This notion is stronger than that of Nash equilibrium as ESS is robust against a deviation of a whole fraction of the population whereas the Nash equilibrium is defined with respect to possible deviations of a single player.

The second basic element of evolutionary games is that of replicator dynamics, which provides the dynamic evolution of the ratio of the population that uses each action, which may or may not converge to an equilibrium. One is interested in the dynamics of the competition and not just in the equilibrium. There are various variants of replicator dynamics, each giving distinct trajectories, that can be justified under appropriate conditions. We note that the ESS concept does not rely on how the dynamics are modeled, and therefore the modeling phase in evolutionary games is often restricted to describing the local interactions between players along with the possible related fitness, and does not include convergence aspects. In this paper we shall study the replicator dynamics as well as the Brown-von Neumann-Nash dynamics. We shall provide some theoretical convergence results which will be illustrated by some examples.

As usual in paradoxes in games [19], the equilibrium is not necessarily monotone increasing in the utilities. This results in two surprising paradoxes in the proposed Hawk-Dove game which we call the Hawk and Dove resource abundance paradox and the initial energy paradox offering hope to better understand the behavior of semi-dynamic power control games over a collision channel.

The structure of the remainder of this paper is as follows. In the next Section, we present the system model and introduce the necessary notation. In Section III, we describe the evolutionary game and address the properties of the fitness. Section IV then defines the concept of Evolutionary Stable Strategy. Next, we compute the (pure and mixed) equilibria considering the case with and without breakdown for the case without recharging in Section $\mathrm{V}$ and extend it to the general model with recharging in Section VI. In Section VII, two dynamic models of the game and their convergence properties are studied. Discussions about paradoxes and numerical illustrations are provided in Section VIII. The paper concludes with a discussion in Section IX and the proofs of the main results are presented in the appendices.

\section{MOdeL}

Consider a sparse network that consists of a large population of mobile stations (MS). Apart from mobile stations, there are also many fixed receivers: throw boxes or relays or base stations which we refer to as base stations (BS) in the remainder. We focus on the case where MSs only transmit when they are in the transmission range of a BS; the situation in which mobiles themselves forward packets of other mobiles is explicitly excluded. As multiple MSs may transmit simultaneously to a BS, interference cannot be avoided. However, assuming that the network is sparse, we do not consider interference between multiple mobiles. If interference occurs, at most two mobile terminals are involved, and the probability of interference between multiple mobile stations is neglected.

This brings us to the pairwise interaction paradigm of evolutionary games. It is assumed that interactions between individuals occur by some random selection process in which pairs of individuals are selected independently. We consider two types of terminals: one type transmits at high power and the other type at low power. We refer to these by Hawks $(H)$ and Doves $(D)$, respectively, thereby referring to the HD game. A mobile user (player) decides which terminal to use, and once this choice is made, he sticks to that choice of terminal for some predetermined time $T$. Considering only pairwise interaction, it is assumed that the sequence of types of terminals with which a given terminal interacts constitutes a sequence of independent and identically distributed (i.i.d.) random variables.

We shall consider two distinct models.

- Model 1 (M1): $T$ is some fix large time, for example two years, which is approximately the expected time until one changes his/her cellular phone. We assume that $T$ does not depend on the type of the phone $(H$ or $D)$.

- Model 2 (M2): $T$ is the time until the mobile runs out of battery power. Note that in this case, $T$ depends on the type. Indeed, as $H$ consumes more energy than $D$, its battery will drain faster.

Models 1 and 2 above have interesting mathematical properties that guarantee the existence of an ESS as described at the end of Section IV which in turn facilitate its computation.

The interference model is characterized by the probabilities for successful transmissions whereas the energy model at hand is characterized by the transition probabilities for the energy levels. We make the following assumptions.

Success probability: Consider a packet transmission of a terminal and let $\delta$ denote the probability that no other terminal interferes with the transmission. If this is not the case, there 
is interference between two terminals and the probability that the packet is transmitted successfully is determined by the types of the terminals involved. Let $p_{s}(i, j)$ denote the success probability of the first terminal assuming that this terminal plays $i$ while the other plays $j(i, j \in\{H, D\})$. We have,

$$
p_{s}(i, j)= \begin{cases}0 & \text { for }(i, j)=(D, H), \\ p_{1} & \text { for }(i, j)=(D, D), \\ 1 & \text { for }(i, j)=(H, D), \\ p_{2} & \text { for }(i, j)=(H, H) .\end{cases}
$$

These success probabilities reflect the fact that Hawks have more chance to transmit successfully than Doves. In particular, the probability of success of a Dove when he fights for a resource against a Hawk $p_{s}(D, H)$ is equal to 0 whereas the probability of success of a Hawk when the Hawk fights for a resource against a Dove $p_{s}(H, D)$ is equal to 1 .

Transition probabilities: If the energy level of an individual is $n$ and its action is $D$, then the energy level decreases to $n-1$ with probability $q_{1}$ or it remains unchanged with probability $q_{2}$. We assume $q_{1}+q_{2} \leq 1$. We do allow $q_{1}+q_{2}<1$, in which case we assume that there is a positive probability of $1-q_{1}-q_{2}$ for a breakdown which does not depend on the energy level. A breakdown is represented as a transition to energy level zero.

Analogously, if the energy level of an individual is $n$ and its action is $H$, then the energy level decreases to $n-1$ with probability $q_{3}$ or it remains unchanged with probability $q_{4}$. We assume that $q_{1}=q_{3}$. As for $D$, we again allow that $q_{3}+q_{4}<1$ in which case we shall have a breakdown probability $1-q_{3}-$ $q_{4}$, a breakdown corresponding to a transition to energy level 0 .

Initial energy level: In the remainder, we assume that a mobile starts at energy level $N_{D}$ or $N_{H}$, depending on the type of mobile. The energy level which take its values in a discrete set $\{0, \ldots, N\}$ represents the number of transmissions the mobile can do. Transmitting with a high power allows for getting a better throughput at the expense of energy loss. As transmission by a Hawk requires more energy, a Dove will be able to transmit more times with the same battery: $N_{D}>N_{H}$. Finally, when the battery is empty, it is immediately replaced.

\section{PROPERTIES OF THE FITNESS}

Both hawks and doves aim to optimize the amount of data that can be send during the lifetime of the battery, hence the fitness is defined as follows

Definition 1. The long term fitness of a mobile is defined as the sum of the expected number of packets sent by that mobile during the lifetime of its battery. We denote by $V(j, i)$ the long term fitness of a mobile, given that it is of type $j$, and that all others are of type $i$, with $i, j \in\{H, D\}$.

Definition 2. Assume that at any time, a fraction $\alpha$ of the mobiles use action $D$, and the rest use $H$. We then denote by $V(j, \alpha)$ the corresponding long term fitness given that the mobile uses $j$. Moreover, let

$$
V(\beta, \alpha)=\beta V(D, \alpha)+(1-\beta) V(H, \alpha)
$$

be the fitness of a terminal that chooses mobile type $D$ (and always uses it) with probability $\beta$, and otherwise chooses type $H$ (with probability $1-\beta$ ).

We shall motivate these definitions at the end of Section IV. We now mention some properties of the fitness.

(i) Throughout, $H$ and $D$ stand both for an action (the power level transmitted at a given time by a given mobile) and a type. The type of a mobile can be interpreted as a state independent pure stationary strategy. Here, a pure stationary strategy is a function that maps states (energy states in our case) to actions.

(ii) Note that the transition probabilities of a user do not depend on the actions of the other users it interacts with. Therefore the total time till a battery drains out is only a function of the mobile's type and not of the actions or types of the other mobiles it interacts with.

(iii) For model M1 (see Section II), $V(\beta, \alpha)$ can be interpreted as the fitness of a player that uses the mixed strategy $\beta$ given that all the rest uses a mixed strategy $\alpha$. A mixed strategy is a random decision of which type of mobile to use ( $H$ or $D)$; Once the (random) decision is made, we assume that the user stays with this terminal during time $T$.

(iv) The interpretation of (iii) is not valid under assumption M2. Indeed, let $T_{i}$ be the time till the battery empties given that it is of type $i$. Assume that all but one mobile use a mixed strategy $\alpha$, i.e., each user chooses to use $D$ until $T_{D}$ with probability $\alpha$. Then the fraction of mobiles that use at a given time an action $D$ is given by

$$
g(\alpha)=\frac{\alpha T_{D}}{\alpha T_{D}+(1-\alpha) T_{H}} .
$$

Conversely, if $\alpha$ stands for the fraction of mobiles that use action $D$ as seen by a MS, then the fraction of mobiles that are of type $D$ (i.e. that use strategy $D$ ) is given by

$$
\frac{\alpha / T_{D}}{\alpha / T_{D}+(1-\alpha) / T_{H}} .
$$

Remark 1. In both models M1 and M2 the time scale between the change of a strategy is much slower that the time scale related to that of a transmission of a packet. Moreover, in all cases this second time scale is much smaller than the time scale of the whole game.

Remark 2. We assume zero-recharging times in the remainder. Nevertheless, for both models M1 and M2 it is possible to include non-zero recharging times. As for model M2, this requires us to account for the fact that only a fraction of the mobiles is active at a time which affects the fraction of mobiles that use a particular strategy at a time. Again, a function $\hat{g}$ can be introduced which relates the fraction $\alpha$ of mobiles that play $D$ to the fraction of active mobiles $\hat{g}(\alpha)$ that play $D$.

\section{Evolutionary Stable Strategies}

\section{A. Nash equilibrium}

As usual, a symmetric strategy $\alpha$ is a Nash equilibrium if no player can do strictly better by a unilateral deviation to some other pure or mixed action $\beta$. 
- For $i=H$ or $i=D, i$ is a pure Nash equilibrium if $V(i, i) \geq V(j, i)$ for $j=H, D$.

- Assuming model M1, we have that $\alpha$ is a mixed Nash equilibrium if $V(\alpha, \alpha) \geq V(\beta, \alpha)$ for all $\beta$.

- Assuming model M2, we have that $\alpha$ is a mixed Nash equilibrium if $V(\alpha, g(\alpha)) \geq V(\beta, g(\alpha))$ for all $\beta$, where $g$ is defined in (2).

An equilibrium is said to be strict if any deviation by any player results in a strictly worse fitness to that player.

\section{B. Definition of a standard evolutionary game}

Suppose that the whole population uses a strategy $j$ and that a small fraction $\epsilon$ (called "mutations") adopts another strategy $i$. Evolutionary forces are expected to select against $i$ if

$$
V(j, \epsilon i+(1-\epsilon) j)>V(i, \epsilon i+(1-\epsilon) j)
$$

Definition 3. A strategy $j$ is said to be an Evolutionary Stable Strategy (ESS) if for every $i \neq j$ there exists some $\bar{\epsilon}_{i}>0$ such that (3) holds for all $\epsilon \in\left(0, \bar{\epsilon}_{i}\right)$.

We shall make use of the following characterization of an ESS [20]:

Theorem 1. A strategy $j$ is an Evolutionary Stable Strategy if and only if $\forall i \neq j$ the following conditions holds:

$$
V(j, j) \geq V(i, j)
$$

and if

$$
V(j, j)=V(i, j) \text { then } V(j, i)>V(i, i)
$$

The first condition says that the ESS is a Nash equilibrium in the game that describes the interaction between two players. Conversely, if $j$ is a strict Nash equilibrium in that game then it is an ESS in the evolutionary game.

The second condition, referred to as "Maynard Smith's second condition", states that if $j$ is a Nash equilibrium but not a strict Nash equilibrium (i.e. the fitness of a deviation $i$ from $j$ does as good as $j$ when the rest of the population uses $j$ ), then $j$ can still be an ESS if it has an advantage in that it can invade the mutants strategy $i$. In other words, in a population where every one uses $i$, a small deviation to $j$ does strictly better than everyone using $i$.

Let $V(i, j)$ denote the expected fitness (utility) for a player when playing a mixed policy $i$ and when the fraction of the population that plays each pure strategy $k$ is given by $j(k)$. The expected fitness is then linear in both $i$ and $j$ and can be written as $i \mathbf{V} j^{T}$ where $\mathbf{V}$ is the matrix whose $m, n$th entry equals $V(m, n)$, and where $i$ (resp. $j$ ) is a row vector whose $m$ th entry is $i(m)$ (resp. $j(m)$ ). Theorem 1 then states that the ESS of an evolutionary game can be characterized by properties of the equilibria of an auxiliary game. In our case this auxiliary game is the matrix game $\mathbf{V}$. Note that not every matrix game has an ESS.

\section{ESS in the semi-dynamic game}

Consider the following two pure strategies of a player (i) always play $D$, and (ii) always play $H$. With some abuse of notation we denote these policies by $D$ and $H$. When writing the long term fitness of players as a function of the system parameters, we shall see that the fitness is linear in $i$ and $j$ whereby $i$ are now probabilities over the strategies $H$ and $D$ and not over the actions $H$ and $D$. This means that a mixed strategy is obtained by tossing a coin, and according to the outcome, the player always uses $D$ or always uses $H$. Notice that if we choose between action $D$ and $H$ with some probability $j$ at each time instant, then the expected fitness need not be linear in $j$. This bilinear form of semidynamic games allows us to apply directly the standard theory of evolutionary games to the semi-dynamic case.

Recall that, even though we assume that each individual mobile always plays the same action, the sequence of actions that are played by the mobiles encountered by some tagged mobile are i.i.d. random variables.

While working with mixed strategies allows for directly applying much of the framework of standard evolutionary games, these policies do not allow for an evolution, as once we perform the initial randomized selection between $D$ and $H$, we shall always stick to that choice. Hence, to combine both the flexibility that allows for evolution together with the linear properties of the auxiliary game (the matrix game introduced above), we assume that each mobile uses mixed policies for some limited time $T$, after which a new choice is made and so on. Recall that $T$ either corresponds to the lifetime of the battery (M2) or to the lifetime of the device (M1).

The definition of $V$ (see Definition 2) is suitable for mixed strategies over an infinite time as well as for the finite horizon framework M1. Recall however that for M2 it should be replaced by $V(\beta, g(\alpha))$ where $g$ is given in (2).

\section{COMPUTING THE EQUILIBRIUM}

Let $V_{n}(i, \alpha)$ denote the expected fitness of a user who plays $i$ and starts at energy level $n, i, j \in\{H, D\}$. In view of this definition we have, $V(D, \alpha)=V_{N_{D}}(D, \alpha)$ and $V(H, \alpha)=$ $V_{N_{H}}(H, \alpha)$. We find the following recursions for $V_{n}(i, \alpha)$,

$$
\begin{aligned}
V_{n}(D, \alpha)= & \delta+(1-\delta) \alpha p_{1}+q_{1} V_{n-1}(D, \alpha) \\
& +q_{2} V_{n}(D, \alpha) \\
V_{n}(H, \alpha)= & \delta+(1-\delta) \alpha+(1-\alpha)(1-\delta) p_{2} \\
& +q_{3} V_{n-1}(H, \alpha)+q_{4} V_{n}(H, \alpha)
\end{aligned}
$$

Equation (6) expresses the total expected fitness of a mobile of type $D$ when starting with $n$ units of energy, till its battery empties. Hence, this equation is composed of two expressions:

(i) The expected fitness corresponding to the current transmission: with probability $\delta$ there is no interference at all so the fitness is one unit. With probability $(1-\delta)$ there is an interaction with another mobile. The fitness equals $p_{1}$ when both mobiles use $D$ which occurs with probability $\alpha$. Otherwise, when the mobile fights for a resource against a Hawk which occurs with probability 
$1-\alpha$, the expected fitness corresponding to the current transmission of a Dove equals 0 .

(ii) The expected fitness collected after the transmission: we first note that with probability $q_{1}$, the energy level after the transmission equals $n-1$, so the expected fitness to go is $q_{1} V_{n-1}(D, \alpha)$. With probability $q_{2}$ the energy level is unchanged so the expected fitness collected after the transmission is $q_{2} V_{n}(D, \alpha)$.

Equation (7) can be explained following similar lines in which the expected fitness corresponding to the current transmission of a Hawk when he fights for a resource against a Dove equals 1 which occurs with probability $\alpha$. This gives the term $(1-\delta) \alpha$ in the the expected fitness $V_{n}(H, \alpha)$ in Eqt. (7).

\section{A. With Breakdown}

Solving the recursions for $q_{1}+q_{2}<1$ and $q_{3}+q_{4}<1$ yields,

$V_{n}(D, \alpha)=\frac{\delta+\alpha p_{1}(1-\delta)}{1-q_{1}-q_{2}}\left(1-\left(\frac{q_{1}}{1-q_{2}}\right)^{n}\right)$,

$V_{n}(H, \alpha)=\frac{\delta+\alpha(1-\delta)+p_{2} \bar{\delta}(1-\alpha)}{1-q_{3}-q_{4}}\left(1-\left(\frac{q_{3}}{1-q_{4}}\right)^{n}\right)$,

whereby we assumed $V_{0}(D, \alpha)=V_{0}(H, \alpha)=0$. That is, no fitness can be collected if the battery is empty.

Lemma 1. Assume that both hawks and doves are subjected to breakdowns $\left(q_{1}+q_{2} \neq 1\right.$ and $\left.q_{3}+q_{4} \neq 1\right)$, we have for $i \in\{D, H\}$,

$$
V(i, \alpha)=\alpha V(i, D)+(1-\alpha) V(i, H),
$$

with,

$$
\begin{aligned}
& V(D, D)=\frac{\delta+p_{1}(1-\delta)}{1-q_{1}-q_{2}}\left(\frac{q_{1}}{1-q_{2}}\right)^{N_{D}} \\
& V(H, D)=\frac{1}{1-q_{3}-q_{4}}\left(1-\left(\frac{q_{3}}{1-q_{4}}\right)^{N_{H}}\right), \\
& V(H, H)=\frac{\delta+p_{2}(1-\delta)}{1-q_{3}-q_{4}}\left(1-\left(\frac{q_{3}}{1-q_{4}}\right)^{N_{H}}\right), \\
& V(D, H)=\frac{\delta}{1-q_{1}-q_{2}}\left(\frac{q_{1}}{1-q_{2}}\right)^{N_{D}}
\end{aligned}
$$

This allows us to express the equilibrium as follows.

Corollary 1. Assuming non-zero breakdown probability, the following holds

(i) $D$ is a pure equilibrium if

$$
\frac{1}{1-q_{3}-q_{4}} \cdot\left(\frac{q_{3}}{1-q_{4}}\right)^{N_{H}}+\frac{\delta+p_{1}(1-\delta)}{1-q_{1}-q_{2}} \cdot\left(\frac{q_{1}}{1-q_{2}}\right)^{N_{D}}>\frac{1}{1-q_{3}-q_{4}}
$$

(ii) $H$ is a pure equilibrium if

$$
\frac{\delta+p_{2}(1-\delta)}{1-q_{3}-q_{4}} \cdot\left(\frac{q_{3}}{1-q_{4}}\right)^{N_{H}}+\frac{\delta}{1-q_{1}-q_{2}} \cdot\left(\frac{q_{1}}{1-q_{2}}\right)^{N_{D}}<\frac{\delta+p_{2}(1-\delta)}{1-q_{3}-q_{4}}
$$

(iii) Let

$$
\alpha^{*}=\frac{\theta \cdot \frac{\delta}{1-q_{1}-q_{2}}-\rho \cdot \frac{\left(\delta+p_{2}(1-\delta)\right)}{1-q_{3}-q_{4}}}{\theta \cdot \frac{\left((1-\delta)\left(1-p_{2}\right)\right)}{1-q_{3}-q_{4}}-\rho \cdot \frac{\left((1-\delta) p_{1}\right)}{1-q_{1}-q_{2}}}
$$

where

$$
\theta=\left(1-\left(\frac{q_{1}}{1-q_{2}}\right)^{N_{D}}\right)
$$

and

$$
\rho=\left(1-\left(\frac{q_{1}}{1-q_{2}}\right)^{N_{H}}\right) .
$$

If $\alpha^{*}$ is in the interior of the unit interval then it is a mixed ESS.

Notice that the existence of the mixed strategy $\alpha^{*}$ is still not insured. Indeed, one must identify conditions on parameters in order to guarantee that $\alpha^{*}$ ranges between 0 and 1.

Lemma 2. The mixed ESS $\alpha^{*}$ is given by

$$
\alpha^{*}=\frac{V(H, H)-V(D, H)}{V(D, D)+V(H, H)-V(H, D)-V(D, H)}
$$

We have the following existence conditions

(i) $V(D, D)>V(H, D)$ and $V(H, H)>V(D, H)$ or

(ii) $V(D, D)>V(H, D), V(H, H)<V(D, H)$ and $|V(H, H)-V(D, H)|>|V(D, D)-V(H, D)|$.

We can therefore compute the value of the Hawk-Dove game. The value of the game measures the payoff obtained when both players follow their optimal strategies.

Corollary 2. The value of the Hawk-Dove game is

$$
V=\frac{|\Delta|}{V(H, H)+V(D, D)-V(H, D)-V(D, H)}
$$

where $|\Delta|$ stands for the determinant of the matrix game $G$ (The detailed analysis can be found in the Appendix).

\section{B. Without Breakdown}

Assume now that hawk and dove are no more subject to breakdown. We have then $q_{1}=1-q_{2}$ and $q_{3}=1-q_{4}$, which yields

$$
\begin{aligned}
& V_{n}(D, \alpha)=\frac{\alpha p_{1}(1-\delta)+\delta}{q_{1}} n \\
& V_{n}(H, \alpha)=\frac{(1-\delta)(1-\alpha) p_{2}+\delta+\alpha(1-\delta)}{q_{3}} n
\end{aligned}
$$

We make the observation that the behavior of the system depends on the ratio $\frac{N_{H}}{N_{D}}$ and not on $N_{D}$ and $N_{H}$ themselves. Let $\gamma=\frac{N_{H}}{N_{D}}$ which we will call initial energy level ratio. This allows us to express the equilibrium as follows 
Lemma 3. In the absence of breakdowns $\left(q_{1}=1-q_{2}\right.$ and $\left.q_{3}=1-q_{4}\right)$, we have for $i \in\{D, H\}$,

$$
V(i, \alpha)=\alpha V(i, D)+(1-\alpha) V(i, H),
$$

with,

$$
\begin{aligned}
V(H, H) & =\frac{\gamma\left((1-\delta) p_{2}+\delta\right)}{q_{3}}, & V(H, D) & =\frac{\gamma}{q_{3}}, \\
V(D, H) & =\frac{\delta}{q_{1}}, & V(D, D) & =\frac{p_{1}(1-\delta)+\delta}{q_{1}} .
\end{aligned}
$$

This gives us the following equilibria.

Corollary 3. In the absence of breakdowns, the following holds

(i) $D$ is a pure equilibrium if

$$
\gamma<\frac{q_{3}\left(p_{1}(1-\delta)+\delta\right)}{q_{1}}
$$

(ii) $H$ is a pure equilibrium if

$$
\gamma>\frac{\delta q_{3}}{\left(p_{2}(1-\delta)+\delta\right) q_{1}}
$$

(iii) Let

$$
\alpha^{*}=\frac{\delta q_{3}-q_{1} \gamma\left(p_{2}(1-\delta)+\delta\right)}{(1-\delta)\left(q_{1} \gamma\left(1-p_{2}\right)-q_{3} p_{1}\right)}
$$

If $\alpha^{*}$ is in the interior of the unit interval then it is a mixed ESS.

In the following corollary, we give a characterization of the variation of the mixed ESS as function of parameters $\delta$ and $\gamma$.

Corollary 4. The mixed ESS in Eqt. (13) is monotonically decreasing function w.r.t $\delta$ if and only if

$$
\gamma<\frac{q_{3} p_{1}}{q_{1}\left(1-p_{2}\right)}
$$

Proof: Deriving the ESS equilibrium in Eqt. (13) with respect to $\delta$, we have

$$
\frac{\partial \alpha^{*}}{\partial \delta}=\frac{-q_{3}+q_{1} \gamma}{(-1+\delta)^{2}\left(-q_{1} \gamma+q_{1} \gamma p_{2}+q_{3} p_{1}\right)}
$$

The last equation shows that $\alpha^{*}$ changes of sign (independently from $\delta$ ) for $\gamma=q_{3} / q_{1}$ and $\gamma=\frac{q_{3} p_{1}}{q_{1}\left(1-p_{2}\right)}$ whereby the first solution is equal to 1 . This completes the proof.

Corollary 4 points out that the variation of the mixed ESS variation w.r.t $\delta$ depends only on the initial energy level ratio $\gamma$ and not on $\delta$ itself. We will make use of this result next in Section VIII when studying some properties of our H-D game.

At the equilibrium, let $\beta=(\beta, 1-\beta)$ and $\underline{\alpha}=(\alpha, 1-\alpha)$ be two row vectors of probability measures over the available actions $D, H$. Let $\mathbf{V}$ be a matrix whose $(i, j)$ th entry is given by $V(i, j)$. In accordance with Definition 2, denote by $V(\beta, \alpha)$ the expected fitness of a player who always plays strategy $D$ with probability $\beta$ and always $H$ with probability $1-\beta$, while the fraction of individuals in the population that play $D$ is $\alpha$. We then make the following key observation regarding the total expected utility.
Lemma 4. The expected utility for a player that chooses to be $D$ with probability $\beta$ given that the fraction of $D$ in the population is $\alpha$ can be written in a vector form as

$$
V(\beta, \alpha)=\underline{\beta} \mathbf{V} \underline{\alpha}^{T} .
$$

It is thus bilinear. It can therefore be interpreted as the expected fitness for a player in an equivalent one shot game (a symmetric static evolutionary game) where the fraction of $D$ in the population is $\alpha$ and where the player chooses $D$ with probability $\beta$. The equilibria given in Lemma 1 and 2 are ESS.

\section{What ABOUt RechaRging?}

Let us now consider an extension of the model so far. In this section, we assume that there is a possibility for the battery to recharge using solar energy or another form of energy harvesting. Formally, we consider the following recursion equations with the extra term

$$
\begin{aligned}
V_{n}(D, \alpha)=( & \delta \\
& \left.+\alpha p_{1}(1-\delta)\right)+q_{1} V_{n-1}(D, \alpha) \\
& +q_{2} V_{n}(D, \alpha)+q_{5} V_{n+1}(D, \alpha) \\
V_{n}(H, \alpha)=( & +(1-\delta) \alpha)+(1-\alpha)(1-\delta) p_{2} \\
& +q_{3} V_{n-1}(H, \alpha)+q_{4} V_{n}(H, \alpha)+q_{6} V_{n+1}(H, \alpha)
\end{aligned}
$$

The amount of energy recharged between two transmissions is assumed to be small: it is less than the energy spent on transmission at high power (Hawk), but could be more than the transmission energy used at a low power transmission (Dove). We therefore adapt the recursion for $D$ and $H$.

\section{A. With Breakdown}

Let $q_{5}$, resp. $q_{6}$, denote the probability that the energy level of the terminal increases during transmission satisfying $q_{5}<$ $q_{1}$ and $q_{6}<q_{3}$. We then investigate the following recursion with the extra term assuming non-zero breakdown probability $\left(q_{1}+q_{2}+q_{5}<1\right.$ and $\left.q_{3}+q_{4}+q_{6}<1\right)$. In what follows, we solve the recursion for $D$, The resolution for $H$ is similar. We have

$$
V_{n}(D, \alpha)=c_{1} r_{1}^{n}+c_{2} r_{2}^{n}+\frac{\delta+\alpha p_{1}(1-\delta)}{1-q_{1}-q_{2}-q_{5}},
$$

where $c_{1}$ and $c_{2}$ are some constant coefficients, and where $r_{1}$ and $r_{2}$ denote the zeros of the quadratic difference equation $f(r)=q_{5} r^{2}+\left(q_{2}-1\right) r+q_{1}$, namely

$$
r_{1}=\frac{1-q_{2}-\sqrt{\left(1-q_{2}\right)^{2}-4 q_{1} q_{5}}}{2 q_{5}}
$$

and

$$
r_{2}=\frac{1-q_{2}+\sqrt{\left(1-q_{2}\right)^{2}-4 q_{1} q_{5}}}{2 q_{5}} .
$$

Note that we have the following asymptotic limit

$$
\lim _{n \rightarrow \infty} V_{n}(D, \alpha)=\frac{\delta+\alpha p_{1}(1-\delta)}{1-q_{1}-q_{2}-q_{5}} .
$$

That is, if battery power is unlimited, the average breakdown time $\left(1-q_{1}-q_{2}-q_{5}\right)^{-1}$ completely determines the fitness. 
Finally, notice that from $f^{\prime \prime}(r)>0, f(0)>0$ and $f(1)<0$, we have $0<r_{1}<1<r_{2}$. Therefore, the coefficient $c_{2}$ in (16) is equal to 0 . To determine $c_{1}$, recall that $V_{0}(D, \alpha)=0$. In view of (16), we finally obtain,

$$
V_{n}(D, \alpha)=\frac{\delta+\alpha p_{1}(1-\delta)}{1-q_{1}-q_{2}-q_{5}}\left(1-r_{1}^{n}\right)
$$

The expected fitness for hawks can be calculated analogously. We obtain the following expression,

$$
V_{n}(H, \alpha)=\frac{(\delta+(1-\delta) \alpha)+(1-\alpha)(1-\delta) p_{2}}{1-q_{3}-q_{4}-q_{6}}\left(1-r_{3}^{n}\right)
$$

with

$$
r_{3}=\frac{1-q_{4}-\sqrt{\left(1-q_{4}\right)^{2}-4 q_{3} q_{6}}}{2 q_{6}} .
$$

Lemma 5. Assume that both Hawks and Doves are subjected to breakdowns $\left(q_{1}+q_{2}+q_{5} \neq 1\right.$ and $\left.q_{3}+q_{4}+q_{6} \neq 1\right)$, we have for $i \in\{D, H\}$,

$$
V(i, \alpha)=\alpha V(i, H)+(1-\alpha) V(i, D),
$$

with,

$$
\begin{aligned}
& V(D, D)=\frac{\delta+p_{1}(1-\delta)}{1-q_{1}-q_{2}-q_{5}}\left(1-r_{1}^{N_{D}}\right), \\
& V(H, D)=\frac{1}{1-q_{3}-q_{4}-q_{6}}\left(1-r_{3}^{N_{H}}\right), \\
& V(H, H)=\frac{\delta+p_{2}(1-\delta)}{1-q_{3}-q_{4}-q_{6}}\left(1-r_{3}^{N_{H}}\right), \\
& V(D, H)=\frac{\delta}{1-q_{1}-q_{2}-q_{5}}\left(1-r_{1}^{N_{D}}\right) .
\end{aligned}
$$

This allows us to express the equilibrium as follows.

Corollary 5. Assuming non-zero breakdown probability, the following holds

(i) $D$ is a pure equilibrium if

$$
\frac{1-r_{1}^{N_{D}}}{1-r_{3}^{N_{H}}}>\left(p_{1}(1-\delta)+\delta\right) \cdot \frac{1-q_{1}-q_{2}-q_{5}}{1-q_{3}-q_{4}-q_{6}}
$$

(ii) $H$ is a pure equilibrium if

$$
\frac{1-r_{3}^{N_{H}}}{1-r_{1}^{N_{D}}}>\frac{\delta}{\delta+p_{2}(1-\delta)} \cdot \frac{1-q_{3}-q_{4}-q_{6}}{1-q_{1}-q_{2}-q_{5}}
$$

(iii) Let $\alpha^{*}=\frac{A}{B}$ where

$$
A=\left(1-r_{3}^{N H}\right) \cdot \frac{\left(\delta+p_{2}(1-\delta)\right)}{1-q_{3}-q_{4}-q_{6}}-\left(1-r_{1}^{N D}\right) \cdot \frac{\delta}{1-q_{1}-q_{2}-q_{5}}
$$

and

$$
B=\left(1-r_{3}^{N H}\right) \frac{\left(\delta-1+p_{2}(1-\delta)\right)}{1-q_{3}-q_{4}-q_{6}}+\left(1-r_{1}^{N D}\right) \frac{\delta+p_{2}(1-\delta)}{1-q_{1}-q_{2}-q_{5}}
$$

If $\alpha^{*}$ is in the interior of the unit interval then it is a mixed ESS.

\section{B. Without Breakdown}

In the absence of breakdowns (i.e., $q_{1}+q_{2}+q 5=1$ and $\left.q_{3}+q_{4}+q_{6}=1\right)$, we get,

$$
\begin{aligned}
V_{n}(D, \alpha) & =\frac{\left(\delta+\alpha p_{1}(1-\delta)\right)}{q_{5}-q_{1}} n \\
V_{n}(H, \alpha) & =\frac{(\delta+(1-\delta) \alpha)+(1-\alpha)(1-\delta) p_{2}}{q_{6}-q_{3}} n
\end{aligned}
$$

In the latter case, we conclude the following:

Lemma 6. In the absence of breakdowns, we have

$V(H, H)=\frac{(1-\delta) p_{2}+\delta}{q_{6}-q_{3}} \gamma, \quad V(H, D)=\frac{1}{q_{6}-q_{3}} \gamma$,

$V(D, H)=\frac{\delta}{q_{5}-q_{1}}, \quad V(D, D)=\frac{p_{1}(1-\delta)+\delta}{q_{5}-q_{1}}$.

This then gives us the following equilibria.

Corollary 6. In the absence of breakdowns, the following holds

(i) $D$ is a pure equilibrium if $\gamma<\left(p_{1}(1-\delta)+\delta\right) \frac{q_{6}-q_{3}}{q_{5}-q_{1}}$.

(ii) $H$ is a pure equilibrium if $\gamma>\delta\left(p_{2}(1-\delta)+\delta\right) \frac{q_{5}-q_{1}}{q_{6}-q_{3}}$.

(iii) Let

$$
\alpha^{*}=\frac{\delta\left(q_{6}-q_{3}\right)-\left(q_{5}-q_{1}\right)\left(p_{2}(1-\delta)+\delta\right) \gamma}{(1-\delta)\left(\left(q_{5}-q_{1}\right)\left(1-p_{2}\right) \gamma-\left(q_{6}-q_{3}\right) p_{1}\right)}
$$

If $\alpha^{*}$ is in the interior of the unit interval then it is a mixed ESS.

Notice that Lemma 2 still holds for the recharging case.

\section{DYNAMICS}

\section{A. Replicator Dynamics}

Evolutionary games study not only equilibrium behavior but also the dynamics of competition. The most frequently used dynamics to describe the evolution of behavior in a population are the replicator dynamics [21]. It describes the evolution of the fraction $x_{i}(t)$ of the population that uses $i$, as function of the time $t$. Consider a static symmetric evolutionary with a set $\Omega=\{H, D\}$ of available actions and let $V(j, \alpha)$ be the fitness for player when playing action $j$ given that the fraction of the population that plays the different actions is given by $\alpha(j), j \in \Omega$.

The fraction of the population that uses action $j$ evolve according to the replicator dynamics as following:

$$
\begin{gathered}
\dot{x}_{j}(t)=x_{j}(t)[U(j, x(t))-\bar{U}(t)] \\
\bar{U}(t)=\sum_{j \in \Omega} x_{j}(t) U(j, x(t))
\end{gathered}
$$

where

Note that summing equation (20) over $H$ and $D$, we get simply

$$
\dot{x}_{H}(t)+\dot{x}_{D}(t)=0
$$

Assume that initially all components of $x(0)$ are positive. It is clear that if $x(t)$ converges to some stationary point $x^{*}$ then $x^{*}$ is an equilibrium. 
The next proposition gives some insight about convergence conditions for the replicator dynamics with respect to the starting point $\alpha_{0}$ when condition (14) is satisfied.

Proposition 1. Assume that at any time, a fraction $\alpha$ of the mobiles use action $D$, and the others use $H$. As long as condition (14) is verified, we have

- For any starting point of the replicator dynamics $\alpha_{0}$ such that $\alpha_{0}<\alpha^{*}$, the replicator dynamic always converges to the pure ESS strategy $H$.

- For any starting point of the replicator dynamics $\alpha_{0}$ such that $\alpha_{0}>\alpha^{*}$, the replicator dynamic always converges to the pure ESS strategy D.

- For any starting point of the replicator dynamics $\alpha_{0}$ such that $\alpha_{0}=\alpha^{*}$, the replicator dynamic always converges to the mixed ESS strategy $\alpha^{*}$.

The proof is given in the Appendix.

\section{B. Brown-von Neumann-Nash dynamics}

Selection dynamics like the replicator dynamics do not allow to introduce new strategies into the population. A strategy may be superior but if it was not present in the initial population, it can never be used in the future. This could be very restrictive for the case of human strategic interaction for instance. To overcome this hurdle, the Brown-von NeumannNash (BNN) dynamics [22] satisfy the fact if there are any (used or unused) strategies that would perform better than the current population average, at least one of them must increase in frequency [23]. In particular, new strategies can enter if they yield better than average payoffs. Berger [24] studied the stability of Nash equilibria under BNN, and Hofbauer [25] showed global stability of completely mixed ESS. We note that Proposition 1 still holds for the BNN dynamics.

\section{RESULTS}

We now illustrate the results of the preceding sections by several numerical examples. We restrict our study to the case without breakdowns and without recharging (the cases with breakdowns and with recharging are similar). We first analyze the behavior of the ESS equilibria obtained in Section V-B as function of the different parameters of the model. Then, we study the convergence behaviour of both dynamics described in Section VII to the ESS equilibria. Unless otherwise stated, for all numerical applications, we assume the following numerical values: $p_{1}=0.3, p_{2}=0.1, q_{1}=q_{3}=0.6$.

From Figure 1 and 2 we can identify two surprising paradoxes in our Hawk-Dove game which we call the Hawk and Dove resource abundance paradox and the initial energy paradox.

\section{A. The Hawk and Dove resource abundance paradox}

Figure 1 shows the effect of $\delta$, the probability of being alone, on the mixed ESS equilibrium for several values of $\gamma$. We identify a paradox which we call the Hawk and Dove resource abundance paradox. First, we see that, as Corollary 4 claims, depending on the initial energy level ratio $\gamma$, we have two different types of behavior of the mixed ESS:

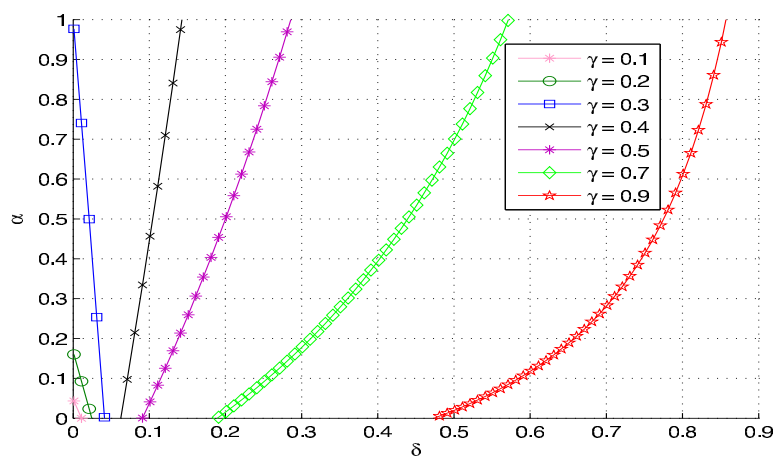

Fig. 1. Variation of the ESS equilibrium $\alpha$ for increasing probability of no interference $\delta$.

- the saturated region: the case where $\gamma<\gamma^{*}$; recall that the threshold $\gamma^{*}=\frac{q_{3} p_{1}}{q_{1}\left(1-p_{2}\right)}=0.33$ was defined in Corollary 4,

- the non-saturated region: the case where $\gamma>\gamma^{*}$.

In the non-saturated region, as intuition would suggest, the proportion of Doves at equilibrium increases for increasing $\delta$, and this happens faster for decreasing $\delta$. This can be explained by the fact that, in the non-saturated region (i.e., low interference), it is more interesting for mobiles to transmit at low power (Dove). This is exactly the opposite for the saturated region where the predominant strategy is Hawk as $\delta$ increases. This is paradoxical since with larger $\delta$, mobiles can get more opportunities to transmit packets successfully at low power. However, one can find an advantage of being aggressive in this region. In fact, in the saturated regime and for a given $\gamma$, mobiles have an incentive to be aggressive (Hawk) since resources are made scarce as the system is highly interfered.

Asymptotically, in a highly interfered system (i.e., for $\delta=0$ ) the value for which all the population is Dove (i.e., $\alpha=1$ ) is given by $\frac{q_{3} p_{1}}{q_{1}}=0.3$ in Figure 1 . This is paradoxical because in a highly interfered system, mobiles should behave aggressively in order to have an opportunity to transmit! Moreover, a small increase in $\delta$ gives more opportunities for mobiles to transmit (more resources are made available within the system). The proportion of Hawk at the equilibrium increases faster.

Figure 1 also depicts the fact that for low values of $\delta$, the ratio $\gamma$ has more impact on the equilibrium. It means that for a large $\delta$, a change on $\gamma$ will not have a big impact on the equilibrium, whereas with a small $\delta$, a tiny change in $\gamma$ will have enormous consequences for the equilibrium. In particular, one observes that the larger the ratio between initial energy states $\left(N_{H}\right.$ and $\left.N_{D}\right)$, the more the equilibrium is sensitive to a small change in $\delta$.

\section{B. The initial energy paradox}

Figure 2 depicts the impact of the initial energy level ratio $\gamma$ on the equilibrium proportion $\alpha$ of Doves for different $\delta$. First note that two different types of behavior for the mixed ESS can be observed. In particular, we find at the left hand side of the figure (where $\alpha$ decreases w.r.t $\gamma$ ) that lower $\gamma$ (i.e., the larger $N_{D}$ for a fixed $N_{H}$ ) yields smaller $\alpha$. This is paradoxical 


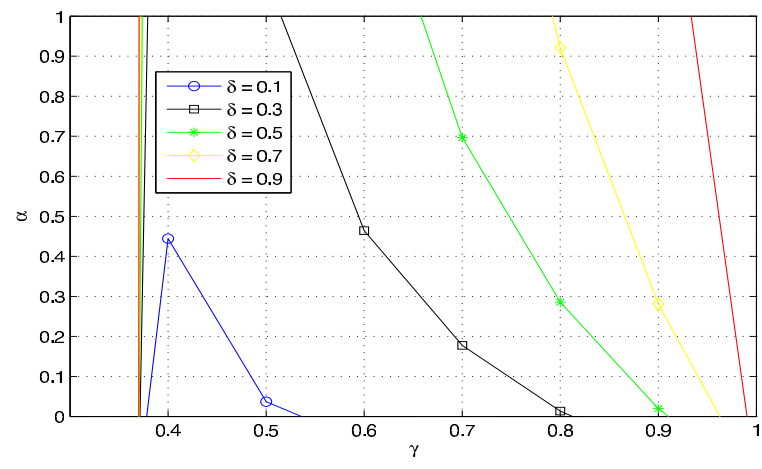

Fig. 2. Variation of the ESS equilibrium $\alpha$ as function of the initial energy level ratio $\gamma$.

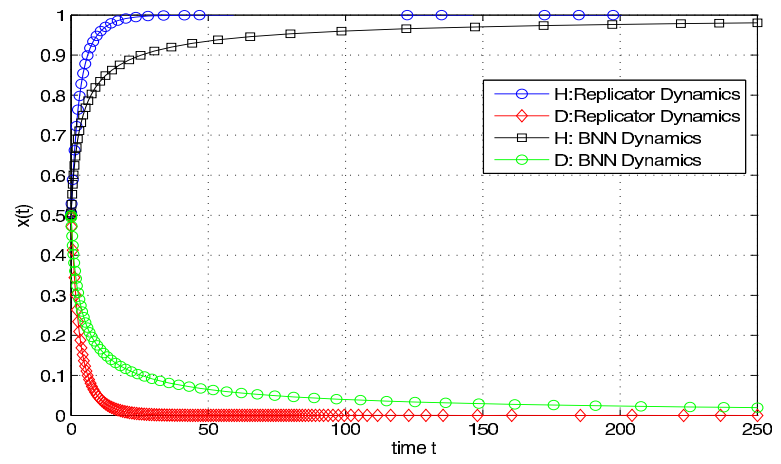

Fig. 3. Convergence of the Replicator Dynamics and the BNN dynamics to the ESS for $N_{H}=1$ and $N_{D}=1$. The pure ESS strategy is Hawk.

because lowering $\gamma$ gives an advantage to the Doves by choosing $D$ a mobile can transmit more packets: both $V(D, D)$ and $V(D, H)$ increase. In the remainder, we will refer to this paradox as the initial energy paradox. As usual in paradoxes in games [19], the equilibrium is not necessarily monotone increasing in the utilities. Some intuition to this behavior is obtained by recalling that a mixed equilibrium $\alpha^{*}$ is characterized by the indifference principle that states that $\alpha^{*}$ is such that the fitness of a player is the same for $D$ and $H$. Now, changing $\gamma$ does not change the fitness of $H$. It increases, but $V(D, H)$ increases more than $V(D, D)$. Therefore to keep each player indifferent between $D$ and $H, \alpha^{*}$ should decrease! Moreover, when the probability $\delta$ of communicating without interference becomes small, one can observe that for some values of the initial energy level ratio $\gamma$, the ESS becomes Dove. This can be explained by the following; The energy level of Doves, respectively Hawks, goes down by 1 with probability $q_{1}$, respectively $q_{3}$. Therefore, the lifetime of Doves and Hawks is equal to $T_{D}=N_{D} / q_{1}$ and $T_{H}=N_{H} / q_{3}$, respectively. In terms of these lifetimes, we have

$$
\alpha^{*}=\frac{\delta T_{D}-\left(p_{2}(1-\delta)+\delta\right) T_{H}}{(1-\delta)\left(\left(1-p_{2}\right) T_{H}-p_{1} T_{D}\right)}
$$

which increases in the neighborhood of $\delta=0$ if $T_{D} \in$ $\left(T_{H}, T_{H}\left(1-p_{2}\right) p_{1}^{-1}\right)$ or $T_{D} \in\left(T_{H}\left(1-p_{2}\right) p_{1}^{-1}, T_{H}\right)$.

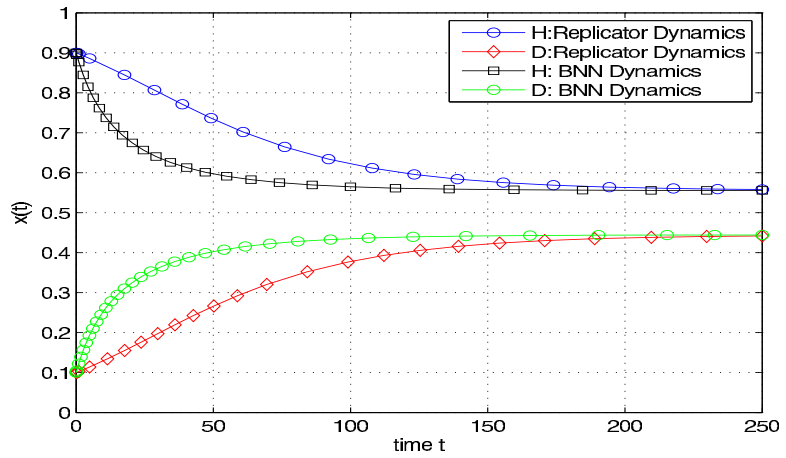

Fig. 4. Convergence of the Replicator Dynamics and the BNN dynamics to the mixed $\mathrm{ESS} \alpha^{*}=0.44$.

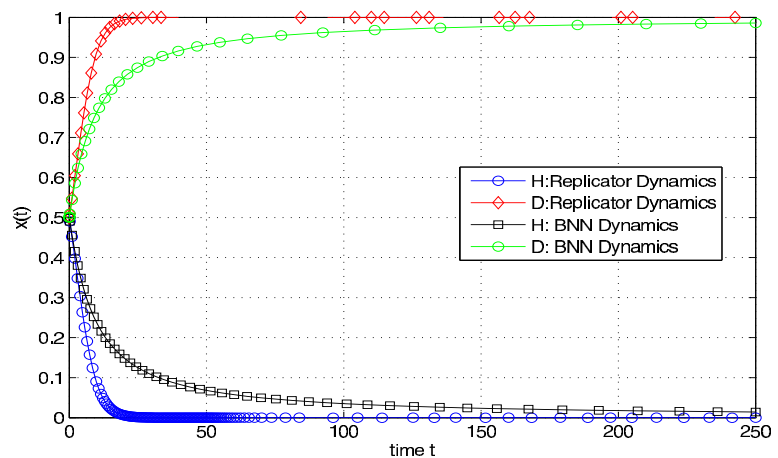

Fig. 5. Convergence of the Replicator Dynamics and the BNN dynamics to the ESS for $N_{H}=1$ and $N_{D}=5$. The pure ESS strategy is Dove.

\section{Dynamics}

We now resort to numerical examples in order to illustrate the impact of different parameters on the convergence to the ESS equilibrium. Figures 3, 4 and 5 validate our theoretical finding about equilibria in Section V-B. In Figure 3, we use the following variables: $N_{H}=1$ and $N_{D}=1$ (i.e., $\gamma=1$ ) and set the initial value of $\alpha$ to $\alpha_{0}=0.5$. It is clearly shown that both dynamics converge to the pure ESS strategy Hawk whereby the replicator dynamics converge faster than BNN dynamics (after 10 iterations, the system reaches the equilibrium). In Figure 4, we then increase the starting energy level for Doves to $N_{D}=$ 2.5 (i.e., $\gamma=0.4$ ) and set the initial value of $\alpha$ to $\alpha_{0}=0.1$. Notice that in this case the initial energy ratio $\gamma$ is larger than the threshold $\gamma^{*}=0.33$ in Corollary 4 which implies from Proposition 1 that the replicator dynamic converges to the mixed ESS ( $\alpha^{*}=0.44$ here). The reader is referred to Proof $\mathrm{C}$ in the Appendix for a detailed analysis of the replicator dynamic convergence conditions. In Figure 5, $N_{D}$ is further increased to 5 (i.e., $\gamma=0.2$ ). Notice that there is convergence to a pure ESS strategy which becomes Dove as a $D$ mobile can transmit more packets with a larger $N_{D}$.

In order to validate the convergence conditions addressed in Proposition 1, we plot the convergence of the replicator dynamics and the BNN dynamic to the ESS for different starting points $\alpha_{0}$. We use the following parameter values: $p_{1}=0.3, p_{2}=0.8, q_{1}=0.6, q_{3}=0.9, \delta=0.1, N_{H}=1$ 


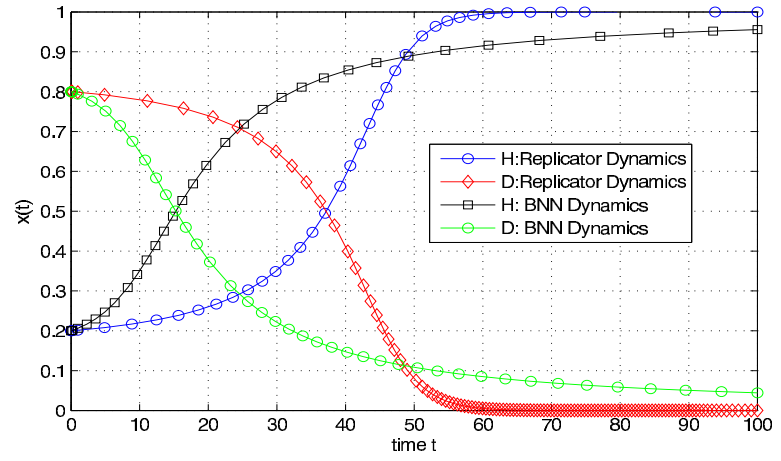

Fig. 6. Convergence of the Replicator Dynamics and the BNN dynamics to the ESS for a starting point $\alpha_{0}=0.8$. The pure ESS strategy is Hawk.

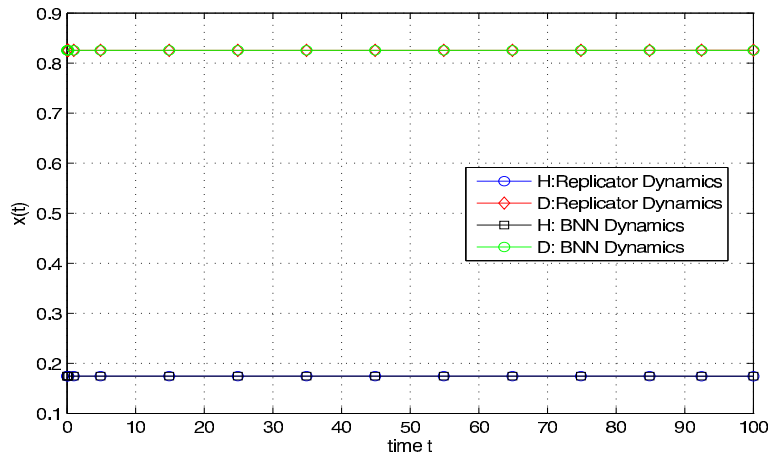

Fig. 7. Convergence of the Replicator Dynamics and the BNN dynamics to the ESS for a starting point $\alpha_{0}=\alpha^{*}=0.825$. Here we converge to the mixed ESS $\alpha^{*}$.

and $N_{D}=2$ (i.e., $\gamma=0.5$ ) which yields an ESS equilibrium $\alpha^{*}=0.825$ (see (13)). We observe from Figure 6 that both dynamics converge to Hawk since we have $\alpha_{0}=0.8<\alpha^{*}$. In Figure 7, we then set the starting point $\alpha_{0}=\alpha^{*}$. Here both dynamics converge to the mixed ESS as pointed out by Proposition 1. Finally, for $\alpha_{0}=0.9>\alpha^{*}$ the dynamics converge to Dove as illustrated in Figure 8. These results validate our theoretical claims on the convergence conditions of the dynamics in Proposition 1.

\section{Discussion AND CONCLUSIONS}

We have studied a semi-dynamic version of the Hawk and Dove game within the framework of evolutionary games. We have identified various surprising paradoxes - namely, the initial energy paradox and the Hawk and Dove resource abundance paradox - which offer insight on how mobiles behave in the framework of semi-dynamic Hawk and Dove game. Moreover, we have studied both replicator and BNN dynamics of the evolutionary H-D game at hand.

The fact that biology gives us tools to analyze and to optimize protocols in communications should not come as a surprise as both autonomous communication networks and populations of animals share decentralized resource allocation problems in general and in particular, issues related to efficient use of energy. Yet we also find several key differences between the initial biological context and ours.

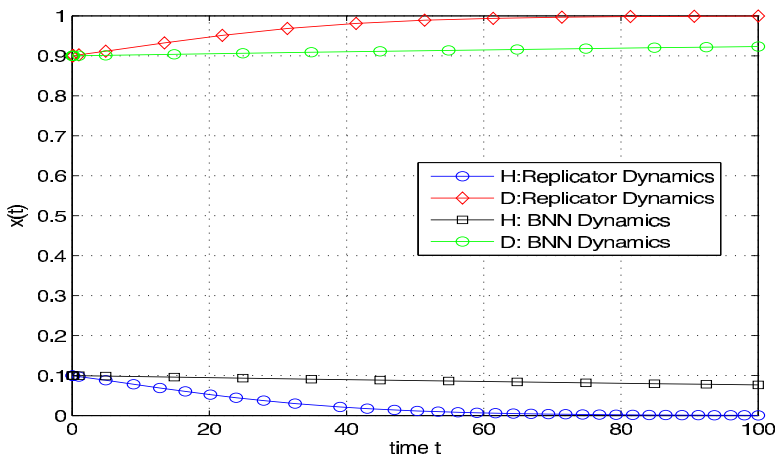

Fig. 8. Convergence of the Replicator Dynamics and the BNN dynamics to the ESS for a starting point $\alpha_{0}=0.9$. The pure ESS strategy is Dove.

- The power control game at hand has the property that the state transition of an individual player only depends on its own action and not on the behavior of other players that he meets. The action of the other player has only an impact on the immediate fitness. In contrast, in the biological context, if an individual is hawk, then the action of the other player not only determines who will get the food but also whether there will be a fight or not. The latter has of course a direct implication on the energy state of an individual.

- We note that in the original H-D game, whenever $\alpha \neq \alpha^{*}$, the replicator dynamic converges to the mixed ESS $\alpha^{*}$ and not to pure strategies. Although this may also hold in the semi-dynamic game (in the biological context), this is not what happens in the power control application where it is shown that the replicator dynamic always converges to a pure ESS strategy (if it exists) when condition (14) is satisfied.

- The classic H-D game is known to be an anti-coordination game. Our H-D game is however a coordination game as long as condition (14) is satisfied. This counters the standard coordination game setup, where all unilateral changes in a strategy lead to either mutual gain or mutual loss. More generally, both Chicken and HawkDove games are anti-coordination games, in which it is mutually beneficial for the players to play different strategies. In this way it can be thought of as the opposite of a coordination game, where playing the same strategy Pareto dominates playing different strategies. The underlying concept is that players use a shared resource. In coordination games, sharing the resource creates a benefit for all: the resource is non-rival, and the shared usage creates positive externalities. In anti-coordination games the resource is rival but non-excludable and sharing comes at a cost (or negative externality).

Next, we raise the question of whether a non-cooperative game is an appropriate framework for this problem. An alternative framework would be the team approach in which several controllers try to optimize jointly some common objective. In many other competing situations in networks, users do adopt a cooperative behavior. An example is the New Reno version of TCP transport protocols, which is probably the mostly used 
protocol. There does not seem to be a trend of trying to take advantage of the gentle behavior of TCP used by others in order to increase one's own throughput by switching to a more aggressive version of TCP. This may be attributed to the fact that the user does not have easy access to the TCP version used on his computer. One may argue that the user does not have access to power control neither. A power control competition between users may still exist through the choice that users do on which mobile terminal to buy.

\section{APPENDIX}

\section{A. Existence of a mixed ESS}

We begin by expressing the fitness at the equilibrium (i.e., for $\alpha^{*}$ ) for both Dove and Hawk, namely

$\alpha^{*} V(D, D)+\left(1-\alpha^{*}\right) V(D, H)=\alpha^{*} V(H, D)+\left(1-\alpha^{*}\right) V(H, H)$

Then we have

$$
\alpha^{*}=\frac{V(H, H)-V(D, H)}{V(D, D)+V(H, H)-V(H, D)-V(D, H)}
$$

We thus obtain the expression of $\alpha^{*}$ as function of the different fitnesses. In order to guarantee the existence of $\alpha^{*}$, the following conditions must be satisfied

(i) To guarantee $\alpha^{*}<1$ we must have $V(D, D)>$ $V(H, D)$

(ii) To guarantee $\alpha^{*}>0$ we have two possibilities; either $V(H, H)>V(D, H)$ or $V(H, H)<V(D, H)$ and $|V(H, H)-V(D, H)|>|V(D, D)-V(H, D)|$.

\section{B. The game's value}

Let us consider the Hawk-Dove game matrix

$$
G=\left(\begin{array}{cc}
V(H, H) & V(H, D) \\
V(D, H) & V(D, D)
\end{array}\right)
$$

The value of this game is

$$
\begin{aligned}
V & =\alpha^{*} V(D, D)+\left(1-\alpha^{*}\right) V(D, H) \\
& =V(D, H)+\alpha^{*}(V(D, D)-V(D, H))
\end{aligned}
$$

Substituting the value of $\alpha^{*}$, see (21), we obtain

$$
\begin{aligned}
V & =V(D, H)+\frac{(V(H, D)-V(H, H))(V(D, D)-V(D, H)}{V(D, H)+V(H, D)-V(H, H)-V(D, D)} \\
& =\frac{|\Delta|}{V(H, H)+V(D, D)-V(H, D)-V(D, H)}
\end{aligned}
$$

where $|\Delta|$ is the determinant of the game matrix $G$.

\section{Replicator Dynamic convergence}

Consider the replicator dynamic given by (20) and replace $x_{D}(t)$ by $\alpha$ the fraction of mobiles using action $D$. We have

$$
\dot{\alpha}=\alpha \cdot \phi(\alpha)
$$

where

$$
\begin{aligned}
& \phi(\alpha)=\alpha V(D, D)+(1-\alpha) V(D, H)-\alpha^{2} V(D, D)- \\
& \alpha(1-\alpha) \cdot V(D, H)-(1-\alpha)^{2} V(H, H)-\alpha(1-\alpha) V(H, D)
\end{aligned}
$$

We want to see if the derivative of $\alpha$ in (22) is monotone on the interval $(0,1)$. Since $\alpha$ is non-negative, the sign of $\dot{\alpha}$ is the same as the sign of $\phi$. Rearranging terms in (23) gives

$$
\begin{aligned}
\phi(\alpha)= & \alpha(1-\alpha) V(D, D)+(1-\alpha)^{2} V(D, H) \\
& -(1-\alpha)^{2} V(H, H)-\alpha(1-\alpha) V(H, D)
\end{aligned}
$$

which yields

$$
\begin{aligned}
\phi(\alpha)= & \alpha(1-\alpha)(V(D, D)-V(H, D)) \\
& +(1-\alpha)^{2}(V(D, H)-V(H, H))
\end{aligned}
$$

Dividing the above equation by $1-\alpha$ for $\alpha \neq 1$, we obtain

$\psi(\alpha)=\alpha(V(D, D)-V(H, D))+(1-\alpha)(V(D, H)-V(H, H))$

Let us now study the sign of $\psi(\cdot)$. Define

$$
\tau=\frac{V(H, H)-V(D, H)}{V(D, D)-V(H, D)}
$$

We make the observation that $\frac{\alpha^{*}}{1-\alpha^{*}}=\tau$, where $\alpha^{*}$ is the mixed ESS in (9) unique solution of $\psi(\alpha)=0$. Now note that the function $\varphi(\alpha)=\frac{\alpha}{1-\alpha}$ is monotone increasing on interval $(0,1)$ which implies that whenever $\alpha>\alpha^{*}$, we have $\varphi(\alpha)>$ $\tau$. Since the scope of this Prop. is limited to condition (14), our Hawk and Dove game yields that $V(D, D)>V(H, D)$ and $V(H, H)>V(D, H)$. The latter inequalities implies that $\psi(\alpha)>0$. We can finally conclude that the derivative of $\alpha$ is always positive, so it can only converge to 1 . In general, we have

- whenever $\alpha>\alpha^{*}, \frac{\alpha}{1-\alpha}>\tau$ yielding $\psi(\alpha)>0$ and thus the derivative of $\alpha$ is always positive, so it can only converge to 1 which corresponds to action $D$.

- whenever $\alpha<\alpha^{*}, \frac{\alpha}{1-\alpha}<\tau$ yielding $\psi(\alpha)<0$ and thus the derivative of $\alpha$ is always negative, so it can only converge to 0 which corresponds to action $H$.

- whenever $\alpha=\alpha^{*}, \frac{\alpha^{*}}{1-\alpha^{*}}=\tau$ yielding $\psi(\alpha)=0$, and thus the derivative of $\alpha$ is always equal to zero, so it can only converge to the mixed $\operatorname{ESS} \alpha^{*}$.

It is noteworthy here that, when condition (14) is not satisfied (and more generally in classical Hawk and Dove games), we have $V(D, D)<V(H, D)$ which implies that whenever $\alpha>\alpha^{*}$, respectively $\alpha<\alpha^{*}$, we have $\psi(\alpha)<0$, respectively $\psi(\alpha)>0$ and thus the derivative of $\alpha$ is always negative, respectively positive. Accordingly, in both cases the replicator dynamic converges to the mixed $\operatorname{ESS} \alpha^{*}$ and not to pure ESS strategies.

\section{REFERENCES}

[1] Eitan Altman, Dieter Fiems, Majed Haddad and Julien Gaillard, "Semi-dynamic Hawk and Dove game, applied to power control", IEEE INFOCOM 2012, Orlando, Florida, USA

[2] J. Maynard Smith, "Game Theory and the Evolution of Fighting", On Evolution (Edinburgh: Edinburgh University Press), pp.8-28, 1972.

[3] H. Tembine, E. Altman, and R. El-Azouzi, "Delayed Evolutionary Game Dynamics applied to the Medium Access Control", Bionetworks 2007, Pisa, Italy.

[4] Y. Hayel, H. Tembine, E. Altman and R. El-Azouzi, "A Markov Decision Evolutionary Game for Individual Energy Management", Annals of the International Society of Dynamic Games, volume 11, pp 313-335, 2011.

[5] Y. Zheng, Z. Feng, "Evolutionary game and resources competition in the Internet", Modern Communication Technologies, SIBCOM, 2001. 
[6] E. Altman, R. El-Azouzi, Y. Hayel and H. Tembine, "The evolution of transport protocols: An evolutionary game perspective", Computer Networks (Elsevier), Volume 53, Issue 10, 14 July 2009, pp 17511759.

[7] R. D. Yates, ”A framework for uplink power control in cellular radio systems", IEEE J. Selected Areas Communications, vol. 13, no. 7, pp. 1341-1347, Sep. 1995

[8] D. J. Goodman and N. B. Mandayam, "Power control for wireless data", IEEE Personal Communications, vol. 7, pp. 48-54, 2000.

[9] N. Bonneau, E. Altman, M. Debbah, and G. Caire, "An Evolutionary Game Perspective to ALOHA with Power Control", 19th International Teletrafc Congress, Beijing, China, Aug. 29-Sep., 2005.

[10] M. Dyer and V. Mohanaraj, "Pairwise-interaction games", In Proceedings of the 38th international colloquim conference on Automata, languages and programming (ICALP'11).

[11] J. Maynard Smith, Evolution and the Theory of Games, Cambridge University Press, 1982.

[12] P. Wiecek, E. Altman and Y. Hayel, "Stochastic state dependent population games in wirelesss communications", Transactions on Autoatic Control, Volume 56 Number 3, pages 492-505, March, 2011.

[13] E. Altman and Y. Hayel, "Markov Decision Evolutionary Games", IEEE Transactions on Automatic Control, vol. 55, Issue 6, June 2010

[14] J. McNamara, S. Merad, E. Collins, "The Hawk-Dove Game as an Average-Cost Problem", Avdances in Applied Probability, Vol. 23, no. 4, pp. 667-682, 1991

[15] E. Altman, J. Gaillard, M. Haddad and P. Wiecek, "Dynamic Hawk and Dove games within flocks of birds", Bionetics, York, England, 2011.

[16] R. Cressman, "Evolutionary Dynamics and Extensive Form Games", MIT Press, 2003.

[17] R. Cressman and A.T. Dash. Density dependence and evolutionary stable strategies. Journal of Theoretical Biology, 126(4):393-406, 1987.

[18] R. Cressman and V. Krivan. The Ideal Free Distribution as an Evolutionarily Stable State in Density-Dependent Populations Games. OIKOS, Vol. 119(8), pp. 1231-1242, August 2010.

[19] E. Altman, Paradigms for Biologically-Inspired Autonomic Networks and Services, The BIONETS Project eBook, available [online]: http: //www.bionets.eu/docs/BIONETS_book.pdf

[20] M. van Veelen and P. Spreij. "Evolution in games with a continuous action space", Economic Theory, 39(3):355-376, 2008.

[21] Carlos P. Roca, Jos A. Cuesta, Angel Snchez, Evolutionary game theory: Temporal and spatial effects beyond replicator dynamics, Physics of Life Reviews, Volume 6, Issue 4, Pages 208-249, December 2009.

[22] G. W. Brown and J. von Neumann, Solutions of games by differential equations, Contributions to the Theory of Games I, volume 24 of Annals of Mathematics Studies, pages 7379, Princeton, 1950.

[23] Josef Hofbauer, Jrg Oechssler, Frank Riedel, Brownvon NeumannNash dynamics, The continuous strategy case, Games and Economic Behavior, Volume 65, Issue 2, March 2009, Pages 406429.

[24] U. Berger, "Best response dynamics and Nash dynamics for games", Dissertation, University of Vienna, 1998.

[25] J. Hofbauer, "From Nash and Brown to Maynard Smith: Equilibria, dynamics and ESS", Selection 1, 81-88, 2000.

\section{ACKNOWLEDGMENT}

The authors would like to thank...

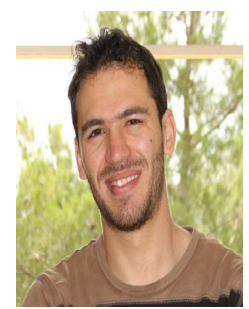

Majed Haddad received his Electrical Engineering diploma in 2004 from the National Engineering School of Tunis (ENIT), Tunisia. He received the Master degree from the University of Nice SophiaAntipolis in France in 2005, and a doctorate in Electrical Engineering from Eurecom Institute in 2008. In 2009, he joined France Telecom R\&D as a post-doctoral Research Fellow. Dr. Haddad is currently pursuing his postdoctoral research at INRIA Sophia-Antipolis in France under an INRIAALCATEL-LUCENT Bell-labs fellowship where his main research interests are in radio resource management, heterogeneous networks, green networks, complex networks and game theory. Majed Haddad has published more than 40 research papers in international conferences, journals, book chapters and patents. He also acts as TPC chair, TPC member and reviewer for various prestigious conferences and journals.

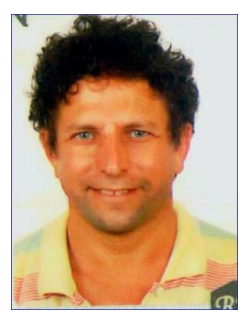

Eitan Altman received the B.Sc. degree in electrical engineering (1984), the B.A. degree in physics (1984) and the Ph.D. degree in electrical engineering (1990), all from the Technion-Israel Institute, Haifa. In (1990) he further received his B.Mus. degree in music composition in Tel-Aviv University. Since 1990, he has been with INRIA (National research institute in informatics and control) in Sophia-Antipolis, France. His current research interests include performance evaluation and control of telecommunication networks and in particular congestion control, wireless communications and networking games. He is in the editorial board of several scientific journals: JEDC, COMNET, DEDS and WICON. He has been the (co)chairman of the program committee of several international conferences and workshops on game theory, networking games and mobile networks.

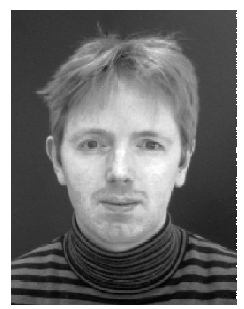

Dieter Fiems is a researcher at the department TELIN (telecommunications and information processing) of Ghent University and a member of the SMACS (Stochastic Modelling and Analysis of Communication Systems) research group since 1998. Dr. Dieter obtained the PhD in engineering from Ghent University in 2004. After his PhD, he has made several longer term research visits to a.o. INRIA-Sophia-Antipolis ( $\mathrm{Fr}$ ), the university of Avignon (Fr) and the university of Petrozavodsk $(\mathrm{Ru})$. Dieter Fiems is currently a postdoctoral fellow with the Research Foundation, Flanders (FWO-Vlaanderen) and part-time professor at Ghent university. His research focusses on stochastic modeling and analysis of communication and production systems.

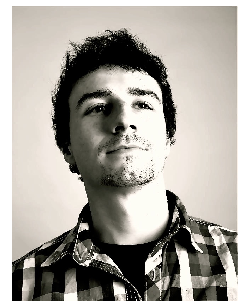

Julien Gaillard has received his BioInformatics and Mathematics diploma in 2011 from the National Institute of Applied Sciences of Lyon (INSA Lyon), France. He is currently doing a PhD thesis at INRIA Sophia-Antipolis on game theory applied to power management problems. He has already published research papers in international conferences (INFOCOM '12, Bionetics '12). 